\title{
Stereoselective Synthesis of Cyercene A and the Placidenes
}

\author{
Guangxin Liang, Aubry K. Miller and Dirk Trauner* \\ Center for New Directions in Organic Synthesis, Department of Chemistry, University of \\ California-Berkeley, Berkeley, California 94720
}

\section{Supporting Information}

Unless otherwise noted, all reagents were purchased from commercial suppliers and used without further purification. Melting points were measured on a Büchi melting point apparatus and are uncorrected. ${ }^{1} \mathrm{H}$ - and ${ }^{13} \mathrm{C}-\mathrm{NMR}$ spectra were recorded on a Bruker DRX 500 or a Bruker AVB 400 spectrometer in $\mathrm{CDCl}_{3}$. Infrared spectra (IR) were obtained on $\mathrm{NaCl}$ plates with an ATI Mattson Gemini FTIR spectrometer. High-resolution mass spectra (HRMS) were obtained on a VG ProSpec Mass Spectrometer using electron impact (EI) at 70 $\mathrm{eV}$.

Unless otherwise noted, all reaction mixtures were magnetically stirred in oven-dried glassware under a blanket of nitrogen or argon. External bath temperatures were used to record all reaction mixture temperatures. Analytical thin layer chromatography (TLC) was carried out on Merck silica gel $60 \mathrm{~F}_{254}$ TLC plates. TLC visualization was accomplished using $254 \mathrm{~nm}$ UV light or charring solutions of $\mathrm{KMnO}_{4}$ and cerric ammonium molybdenate. Flash chromatography was performed on ICN siliTech 32-63 D $60 \AA$ silica gel according to the procedure of Still. ${ }^{1}$

Tetrahydrofuran (THF) and dichloromethane $\left(\mathrm{CH}_{2} \mathrm{Cl}_{2}\right)$ were dried according to the procedure described by Bergman. ${ }^{2}$ Benzene was distilled from $\mathrm{CaH}_{2}$ immediately prior to use. DMF was dried over $4 \AA$ molecular sieves. $n$-Butyl lithium was titrated using diphenylacetic acid in THF. CuI was purified by precipitation from hot aqueous NaI. Extracts were dried over anhydrous $\mathrm{MgSO}_{4}$ and solvents were removed with a rotary evaporator at aspirator pressure.

The preparation of compounds 10 and 11, 15 and 18, and the Stille crossing couplings leading to the natural products followed the same general procedures individually. Representative procedures are demonstrated below.

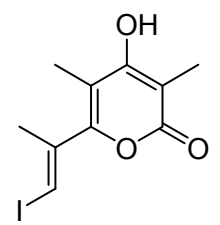

15

4-hydroxy-6-((E)-2-iodo-1-methyl-vinyl)-3,5-dimethyl-pyran-2-one (15): To a suspension of $\mathrm{NaH}$ (475 mg, $11.9 \mathrm{mmol}, 60 \%$ in oil) in THF $(75 \mathrm{~mL})$ was added 2-methyl-3oxopentanoic acid ethyl ester $(1.60 \mathrm{~g}, 11.1 \mathrm{mmol})$ at $0{ }^{\circ} \mathrm{C}$ under argon. After $10 \mathrm{~min}$, a solution of $n$-BuLi $(4.80 \mathrm{~mL}, 12.0 \mathrm{mmol}, 2.5 \mathrm{M}$ in hexanes) was added. After $10 \mathrm{~min}$, a solution of $13(1.45 \mathrm{~g}, 7.40 \mathrm{mmol})$ in THF $(5 \mathrm{~mL})$ was added. After $1.5 \mathrm{~h}$, the reaction mixture was quenched with saturated aqueous $\mathrm{NH}_{4} \mathrm{Cl}(100 \mathrm{~mL})$ and the two layers were 
separated. The aqueous layer was extracted with $\mathrm{Et}_{2} \mathrm{O}(2 \times 40 \mathrm{~mL})$ and the combined organic layers were dried $\left(\mathrm{MgSO}_{4}\right)$, filtered, and concentrated in vacuo. The resulting oil was purified by column chromatography (30\% EtOAc in hexanes) to provide a mixture of diastereomers that was taken on without characterization. To a solution of this mixture in $\mathrm{CH}_{2} \mathrm{Cl}_{2}$ taken directly from a squirt bottle $(75 \mathrm{~mL})$ was added the Dess-Martin periodinane $(3.80 \mathrm{~g}, 8.96$ $\mathrm{mmol})$ at $0{ }^{\circ} \mathrm{C}$ open to the air. After addition, the reaction mixture was allowed to warm to $\mathrm{rt}$. After $1 \mathrm{~h}$, a mixture of 1:1:1 $\mathrm{H}_{2} \mathrm{O}$ : saturated aqueous $\mathrm{NaHCO}_{3}$ :saturated aqueous $\mathrm{Na}_{2} \mathrm{~S}_{2} \mathrm{O}_{3}(100$ $\mathrm{mL}$ ) was added and the two layers were separated. The aqueous layer was extracted with $\mathrm{CH}_{2} \mathrm{Cl}_{2}(2 \times 40 \mathrm{~mL})$ and the combined organic layers were dried $\left(\mathrm{MgSO}_{4}\right)$, filtered, concentrated in vacuo. The resulting oil was purified by column chromatography (20\% EtOAc in hexanes) to provide a mixture of diastereomers that was taken on without characterization. To a solution of this diastereomeric mixture in benzene $(50 \mathrm{~mL})$ was added 1,8-diazobicyclo[5.4.0] undec-7-ene $(1.10 \mathrm{~mL}, 7.35 \mathrm{mmol})$. The resulting mixture was heated to $70{ }^{\circ} \mathrm{C}$ under argon for $1 \mathrm{~h}$. The reaction mixture was concentrated in vacuo and purified by column chromatography ( $4 \% \mathrm{MeOH}, 0.1 \% \mathrm{AcOH}$ in $\mathrm{CH}_{2} \mathrm{Cl}_{2}$ ) to provide $1.20 \mathrm{~g}$ of $15(53 \%)$ as a white solid: $\mathrm{mp} 164-166{ }^{\circ} \mathrm{C}$; $\mathrm{R}_{f} 0.34\left(5 \% \mathrm{MeOH}\right.$ in $\left.\mathrm{CH}_{2} \mathrm{Cl}_{2}\right)$; IR (thin film) 3070 (br), 1661, $1556 \mathrm{~cm}^{-1} ;{ }^{1} \mathrm{H}$ NMR $(500 \mathrm{MHz}): \delta_{\mathrm{H}} 6.66(\mathrm{~s}, 1 \mathrm{H}), 5.63(\mathrm{~s}, 1 \mathrm{H}), 2.11(\mathrm{~s}, 3 \mathrm{H}), 2.00(\mathrm{~s}, 6$ $\mathrm{H}) ;{ }^{13} \mathrm{C}$ NMR $(100 \mathrm{MHz}): \delta_{\mathrm{C}} 165.9,165.8,154.8,139.7,109.0,100.2,88.8,23.1,11.8,9.1$; HRMS calcd for $\mathrm{C}_{10} \mathrm{H}_{11} \mathrm{IO}_{3}\left(\mathrm{M}^{+}\right)$: 305.9753 ; found: 305.9755 .

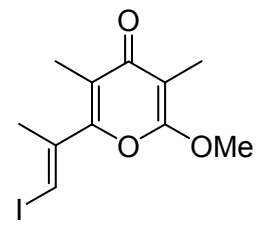

10

2-((E)-2-iodo-1-methyl-vinyl)-6-methoxy-3,5-dimethyl-pyran-4-one (10): To a suspension of $11(890 \mathrm{mg}, 2.91 \mathrm{mmol})$ in $\mathrm{CH}_{2} \mathrm{Cl}_{2}(30 \mathrm{~mL})$ was added $\mathrm{MeOSO}_{2} \mathrm{~F}(1.20 \mathrm{~mL}, 15.0 \mathrm{mmol})$ at $0{ }^{\circ} \mathrm{C}$ under argon. After the addition was complete the mixture was allowed to warm to rt. After $12 \mathrm{~h}$, the reaction mixture was concentrated in vacuo taking great care to avoid exposure to the volatile and highly toxic $\mathrm{MeOSO}_{2} \mathrm{~F}$. The residue was dissolved in $\mathrm{CH}_{2} \mathrm{Cl}_{2}$ and concentrated three more times to remove all $\mathrm{MeOSO}_{2} \mathrm{~F}$. This residue was then taken up in $\mathrm{CH}_{2} \mathrm{Cl}_{2}(25 \mathrm{~mL})$ and $1 \mathrm{~N} \mathrm{NaOH}(50 \mathrm{~mL})$. The two layers were separated and the aqueous layer was extracted with $\mathrm{CH}_{2} \mathrm{Cl}_{2}(2 \times 25 \mathrm{~mL})$. The combined organics were dried $\left(\mathrm{MgSO}_{4}\right)$, filtered, and concentrated in vacuo to provide $888 \mathrm{mg}$ of $\mathbf{1 0}(95 \%)$ as a white solid: $\mathrm{mp}$ 90-93 ${ }^{\circ} \mathrm{C} ; \mathrm{R}_{f} 0.36$ (40\% EtOAc in hexanes); IR (thin film) 1655, 1611, $1590 \mathrm{~cm}^{-1} ;{ }^{1} \mathrm{H}$ NMR (500 $\mathrm{MHz}): \delta_{\mathrm{H}} 6.72(\mathrm{~s}, 1 \mathrm{H}), 3.93(\mathrm{~s}, 3 \mathrm{H}), 2.11(\mathrm{~s}, 3 \mathrm{H}), 1.95(\mathrm{~s}, 3 \mathrm{H}), 1.82(\mathrm{~s}, 3 \mathrm{H}) ;{ }^{13} \mathrm{C}$ NMR $(100$ $\mathrm{MHz}): \delta_{\mathrm{C}} 181.0,161.9,154.6,139.3,119.2,99.9,89.5,55.6,22.9,11.9,7.1$; HRMS calcd for $\mathrm{C}_{11} \mathrm{H}_{13} \mathrm{IO}_{3}\left(\mathrm{M}^{+}\right)$: 319.9909; found: 319.9912 . 


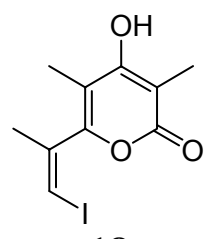

18

\section{4-hydroxy-6-((Z)-2-iodo-1-methyl-vinyl)-3,5-dimethyl-pyran-2-one (18)}

White solid. mp 193.0-194.5 ${ }^{\circ} \mathrm{C}$; $\mathrm{R}_{f} 0.21$ (33\% EtOAc in hex); IR (thin film) 3093(br), 1665, 1611, $1561 \mathrm{~cm}^{-1} ;{ }^{1} \mathrm{H}$ NMR $\left(400 \mathrm{MHz}, \mathrm{CD}_{3} \mathrm{OD}\right) \delta_{\mathrm{H}} 6.66(\mathrm{~s}, 1 \mathrm{H}), 2.01(\mathrm{~s}, 3 \mathrm{H}), 1.89(\mathrm{~s}, 3 \mathrm{H})$, $1.86(\mathrm{~s}, 3 \mathrm{H}) ;{ }^{13} \mathrm{C}$ NMR $\left(100 \mathrm{MHz}, \mathrm{CD}_{3} \mathrm{OD}\right) \delta_{\mathrm{C}} 168.2,167.6,157 ., 142.4,110.1,100.4,83.6$, 23.4, 11.2, 9.2; HRMS calcd for $\mathrm{C}_{10} \mathrm{H}_{11} \mathrm{IO}_{3}\left(\mathrm{M}^{+}\right)$: 305.9753; found: 305.9750 .

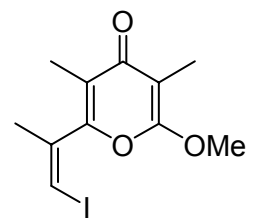

11

\section{2-((Z)-2-iodo-1-methyl-vinyl)-6-methoxy-3,5-dimethyl-pyran-4-one (11)}

Colorless oil. $\mathrm{R}_{f} 0.20$ (25\% EtOAc in hexanes); IR (thin film) $1663,1612,1586 \mathrm{~cm}^{-1} ;{ }^{1} \mathrm{H}$ NMR $(400 \mathrm{MHz}) \delta_{\mathrm{H}} 6.37(\mathrm{q}, J=1.5 \mathrm{~Hz}, 1 \mathrm{H}), 3.94(\mathrm{~s}, 3 \mathrm{H}), 2.08(\mathrm{~d}, J=1.5 \mathrm{~Hz}, 3 \mathrm{H}), 1.88(\mathrm{~s}$, $3 \mathrm{H}), 1.85(\mathrm{~s}, 3 \mathrm{H}) ;{ }^{13} \mathrm{C} \mathrm{NMR}(100 \mathrm{MHz}) \delta_{\mathrm{C}} 180.7,162.4,155.8,140.4,118.9,100.0,83$., 55.6, 23.5, 10.6, 6.9; HRMS calcd for $\mathrm{C}_{11} \mathrm{H}_{13} \mathrm{IO}_{3}\left(\mathrm{M}^{+}\right)$: 319.9909 ; found: 319.9913 .

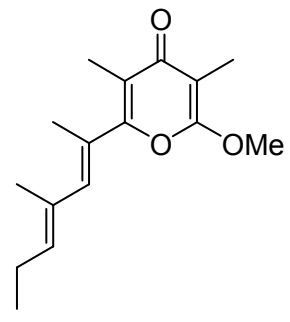

cyercene A (1) A mixture of $\mathbf{1 0}(20.5 \mathrm{mg}, 64 \mu \mathrm{mol}), \mathbf{1 9}(21.3 \mathrm{mg}, 91 \mu \mathrm{mol}), \mathrm{Pd}\left(\mathrm{PPh}_{3}\right)_{4}(4.3$ $\mathrm{mg}, 3.7 \mu \mathrm{mol}), \mathrm{CuI}(1.9 \mathrm{mg}, 10 \mu \mathrm{mol})$ and CsF $(24.1 \mathrm{mg}, 0.16 \mathrm{mmol})$ in DMF $(0.5 \mathrm{~mL})$ was heated to $45^{\circ} \mathrm{C}$ under argon for $1.5 \mathrm{~h}$. The reaction mixture was taken up in $\mathrm{H}_{2} \mathrm{O}(10 \mathrm{~mL})$ and $\mathrm{Et}_{2} \mathrm{O}(3 \mathrm{~mL})$ and the two layers were separated. The aqueous layer was extracted with $\mathrm{Et}_{2} \mathrm{O}(2$ x $3 \mathrm{~mL})$ and the combined organic layers were then washed with $\mathrm{H}_{2} \mathrm{O}(5 \mathrm{~mL})$ and brine $(5$ $\mathrm{mL})$. The organic layer was dried $\left(\mathrm{MgSO}_{4}\right)$, filtered, concentrated in vacuo and purified by column chromatography (30\% EtOAc in hexanes) to provide $10.8 \mathrm{mg}(64 \%)$ of Cyercene A (1) as a white solid: $\mathrm{mp} 52-55{ }^{\circ} \mathrm{C}$; $\mathrm{R}_{f} 0.39$ (40\% EtOAc in hexanes); IR (thin film) 2960, 2927, 1658, 1612, $1600 \mathrm{~cm}^{-1} ;{ }^{1} \mathrm{H}$ NMR $(500 \mathrm{MHz}) \delta_{\mathrm{H}} 6.10(\mathrm{~s}, 1 \mathrm{H}), 5.52(\operatorname{app~t}, J=17.2 \mathrm{~Hz}$, $1 \mathrm{H}), 3.95$ (s, $3 \mathrm{H}), 2.15$ (app quint, $J=17.4 \mathrm{~Hz}, 2 \mathrm{H}), 2.06(\mathrm{~d}, J=1.3 \mathrm{~Hz}, 3 \mathrm{H}), 2.01$ (s, $3 \mathrm{H})$, $1.86(\mathrm{~s}, 3 \mathrm{H}), 1.84(\mathrm{~s}, 3 \mathrm{H}), 1.03(\mathrm{t}, J=17.5 \mathrm{~Hz}, 3 \mathrm{H}) ;{ }^{13} \mathrm{C}(125 \mathrm{MHz}): \delta_{\mathrm{C}} 181.8,162.1,159.3$, 
139.7, 136.1, 131.2, 125.6, 118.0, 99.4, 55.4, 21.8, 16.6, 16.3, 14.1, 12.1, 7.1; HRMS calcd for $\mathrm{C}_{16} \mathrm{H}_{22} \mathrm{O}_{3}\left(\mathrm{M}^{+}\right)$: 262.1569; found: 262.1566 .

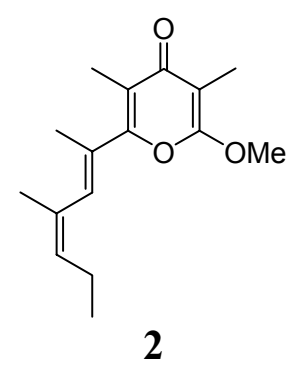

\section{placidene-A (2)}

White solid. mp: 47.0-48.0 ${ }^{\circ} \mathrm{C}$; $\mathrm{R}_{f} 0.33$ (25\% EtOAc in hexanes); IR 2961, 2932, 2874, 1656, $1614,1597 \mathrm{~cm}^{-1} ;{ }^{1} \mathrm{H}$ NMR $(400 \mathrm{MHz}) \delta_{\mathrm{H}} 6.18(\mathrm{~s}, 1 \mathrm{H}), 5.40(\mathrm{t}, J=7.2 \mathrm{~Hz}, 1 \mathrm{H}), 3.97(\mathrm{~s}, 3 \mathrm{H})$, 2.05 (s, $3 \mathrm{H}), 1.99$ (m, $2 \mathrm{H}), 1.91$ (s, $3 \mathrm{H}), 1.88$ (s, $3 \mathrm{H}), 1.85$ (s, $3 \mathrm{H}), 0.98$ (t, $J=7.5 \mathrm{~Hz}, 3 \mathrm{H})$; ${ }^{13} \mathrm{C}$ NMR $(100 \mathrm{MHz}) \delta_{\mathrm{C}} 181.5,161.9,158.3,135.2,132.4,130.3,128.0,118.0,99.3,55.2$, 23.2, 22.7, 16.1, 14.0, 12.0, 6.9; HRMS calcd for $\mathrm{C}_{16} \mathrm{H}_{22} \mathrm{O}_{3}\left(\mathrm{M}^{+}\right)$: 262.1569; found: 262.1570 .

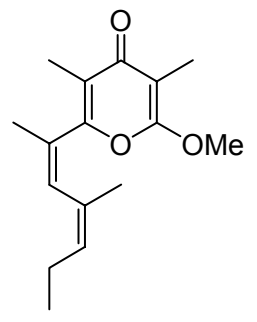

3

\section{isoplacidene-A (3)}

White solid. mp: 68.0-69.0 ${ }^{\circ} \mathrm{C}$; $\mathrm{R}_{f} 0.34$ (25\% EtOAc in hexanes); IR (thin film) 2961, 2926, 2873, 1661, 1613, $1594 \mathrm{~cm}^{-1}$; ${ }^{1} \mathrm{H}$ NMR $(400 \mathrm{MHz}) \delta_{\mathrm{H}} 6.42(\mathrm{~s}, 1 \mathrm{H}), 5.31(\mathrm{t}, J=7.2 \mathrm{~Hz}, 1 \mathrm{H})$, 3.94 (s, $3 \mathrm{H}), 2.08-1.95$ (m, $5 \mathrm{H}), 1.87$ (s, $3 \mathrm{H}), 1.83$ (s, $3 \mathrm{H}), 1.57$ (s, $3 \mathrm{H}), 0.94$ (t, $J=7.4 \mathrm{~Hz}$, $3 \mathrm{H}) ;{ }^{13} \mathrm{C}$ NMR $(100 \mathrm{MHz}) \delta_{\mathrm{C}} 180.8,162.4,156.3,134.8,132.1,130.3,126.8,119.5,99.7$, 55.4, 23.1, 21.8, 21.7, 14.1, 10.8, 6.9; HRMS calcd for $\mathrm{C}_{16} \mathrm{H}_{22} \mathrm{O}_{3}\left(\mathrm{M}^{+}\right)$: 262.1569; found: 262.1566 .

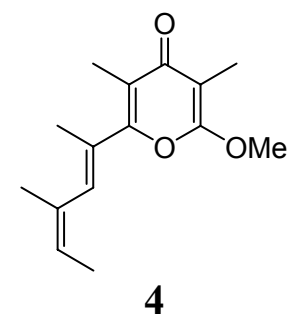

placidene-B (4)

White solid. mp: 76.0-77.0 ${ }^{\circ} \mathrm{C}$; $\mathrm{R}_{f} 0.30$ (25\% EtOAc in hexanes); IR 2957, 2921, 1656, 1610, $1596 \mathrm{~cm}^{-1} ;{ }^{1} \mathrm{H}$ NMR $(400 \mathrm{MHz}) \delta_{\mathrm{H}} 6.15(\mathrm{~s}, 1 \mathrm{H}), 5.49(\mathrm{q}, J=6.9 \mathrm{~Hz}, 1 \mathrm{H}), 3.98(\mathrm{~s}, 3 \mathrm{H}), 2.06$ 
$(\mathrm{s}, 3 \mathrm{H}), 1.90(\mathrm{~s}, 3 \mathrm{H}), 1.88(\mathrm{~s}, 3 \mathrm{H}), 1.85(\mathrm{~s}, 3 \mathrm{H}), 1.61(\mathrm{~d}, J=6.9 \mathrm{~Hz}, 3 \mathrm{H}) ;{ }^{13} \mathrm{C}$ NMR $(100$ MHz) $\delta_{\mathrm{C}} 181.5,161.9,158.3,135.0,131.9,128.1,124.5,118.1,99.4,55.3,23.2,16.1,15.1$, 12.1, 6.9; HRMS calcd for $\mathrm{C}_{15} \mathrm{H}_{20} \mathrm{O}_{3}\left(\mathrm{M}^{+}\right)$: 248.1412; found: 248.1416 .

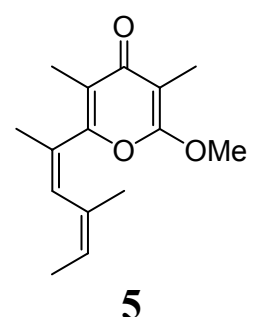

\section{isoplacidene- $B(5)$}

White solid. mp: 80.0-81.0 ${ }^{\circ} \mathrm{C}$; $\mathrm{R}_{f} 0.31$ (25\% EtOAc in hexanes); IR (thin film) 2954, 2917, $1655,1611,1591 \mathrm{~cm}^{-1} ;{ }^{1} \mathrm{H}$ NMR $(400 \mathrm{MHz}) \delta_{\mathrm{H}} 6.41(\mathrm{~s}, 1 \mathrm{H}), 5.40(\mathrm{q}, J=7.0 \mathrm{~Hz}, 1 \mathrm{H}), 3.94$ (s, $3 \mathrm{H}), 2.06(\mathrm{~s}, 3 \mathrm{H}), 1.87(\mathrm{~s}, 3 \mathrm{H}), 1.83(\mathrm{~s}, 3 \mathrm{H}), 1.61(\mathrm{~d}, J=7.0 \mathrm{~Hz}, 3 \mathrm{H}), 1.57(\mathrm{~s}, 3 \mathrm{H}) ;{ }^{13} \mathrm{C}$ NMR $(100 \mathrm{MHz}) \delta_{\mathrm{C}} 180.9,162.4,156.3,132.0,131.8,126.8,119.5,99.7,55.4,23.1,21.8$, 14.1, 10.8, 6.9 (The missing carbon signal is assumed to be due to the isochronicity of two quaternary carbons. By correlation with isoplacidene-A, this appears to be the $131.8 \mathrm{ppm}$ signal. An APT spectrum is attached in addition to the normal ${ }^{13} \mathrm{C}$ spectrum.); HRMS calcd for $\mathrm{C}_{15} \mathrm{H}_{20} \mathrm{O}_{3}\left(\mathrm{M}^{+}\right)$: 248.1412; found: 248.1408 .

\footnotetext{
${ }^{1}$ Still, W. C.; Kahn, M.; Mitra, A. J. Org. Chem. 1978, 43, 2923-2925.

${ }^{2}$ Alaimo, P. J.; Peters, D. W.;Arnold, J.; Bergman, R. G. J. Chem. Ed. 2001, 78, 64.
} 


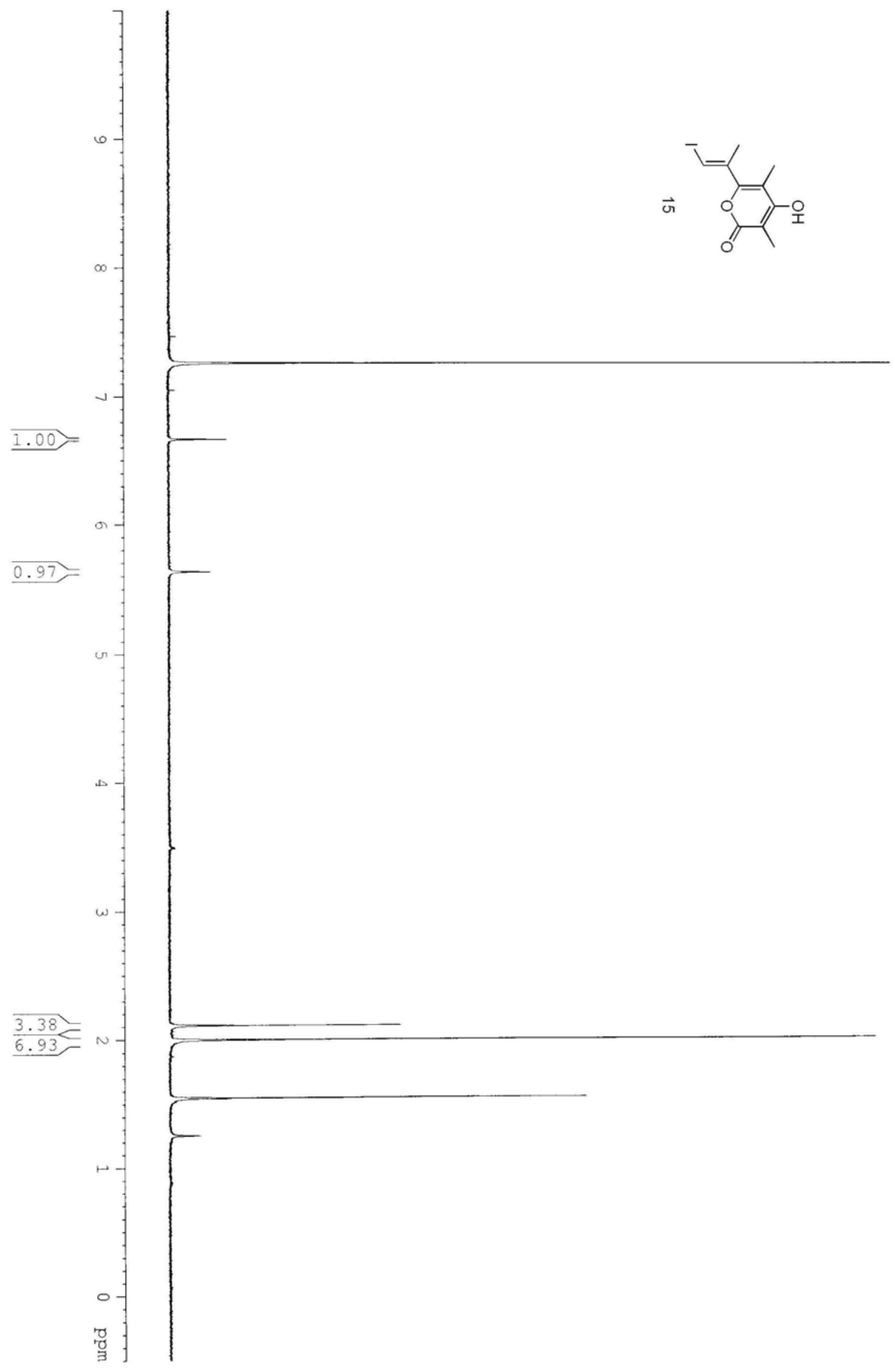




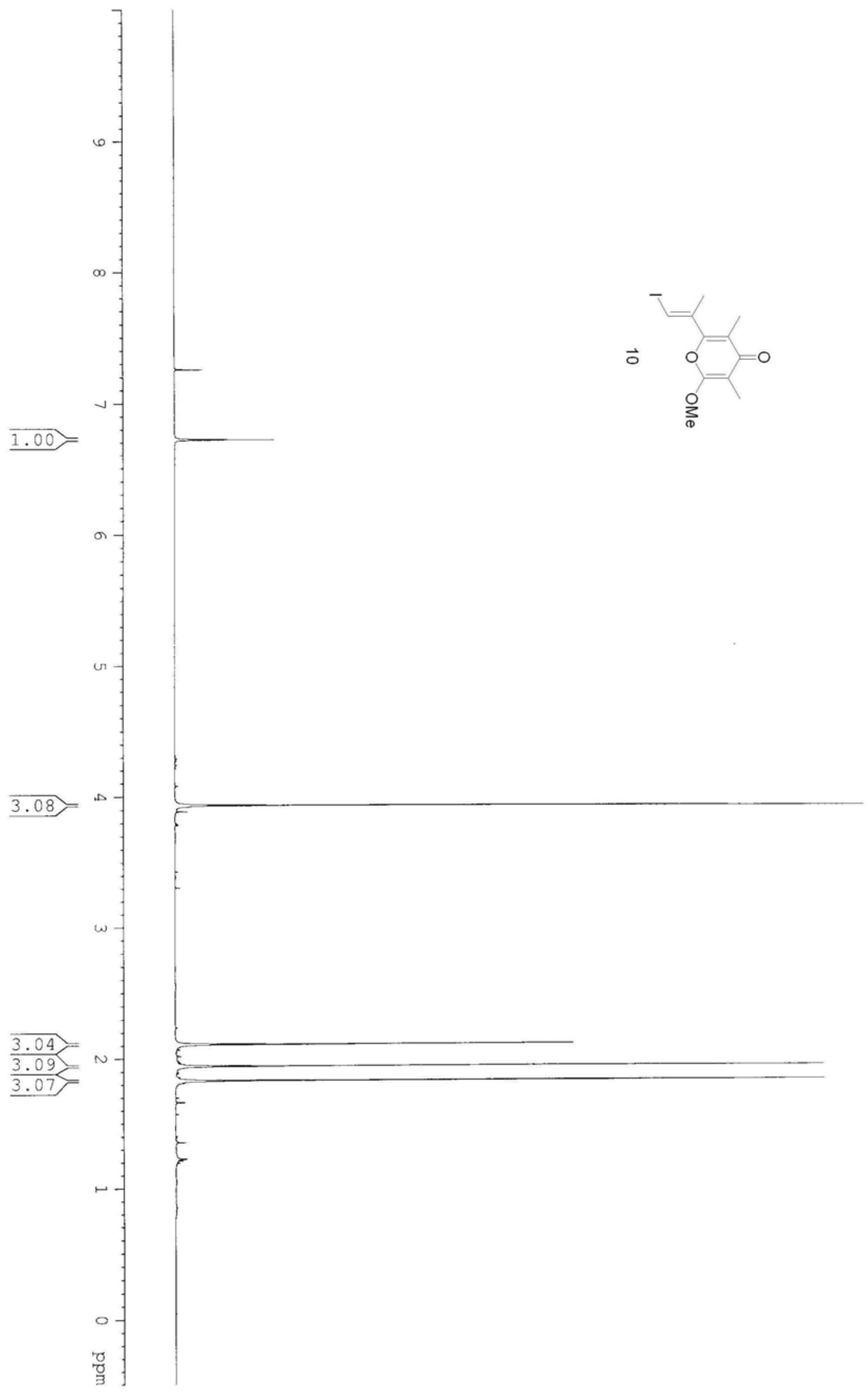




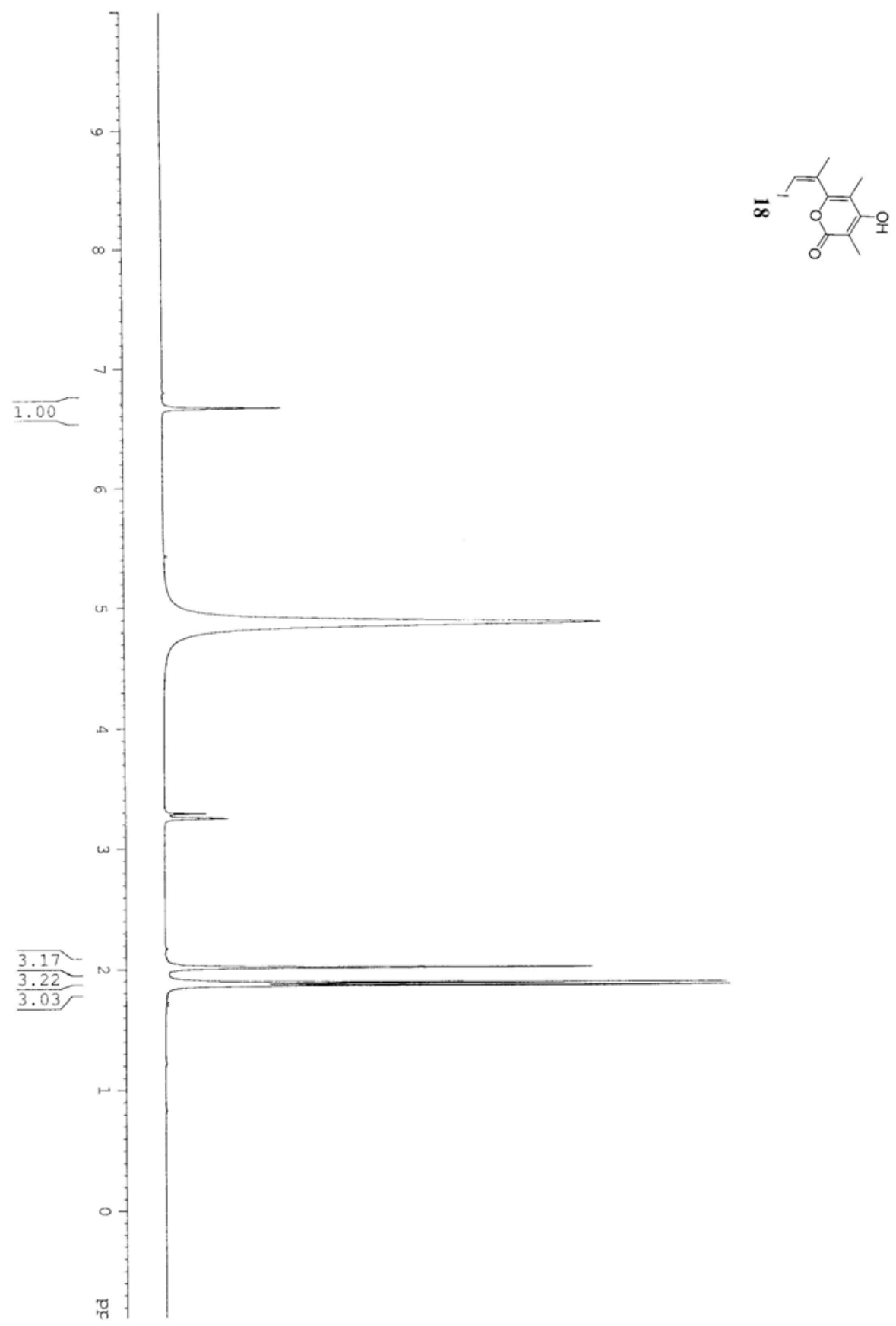




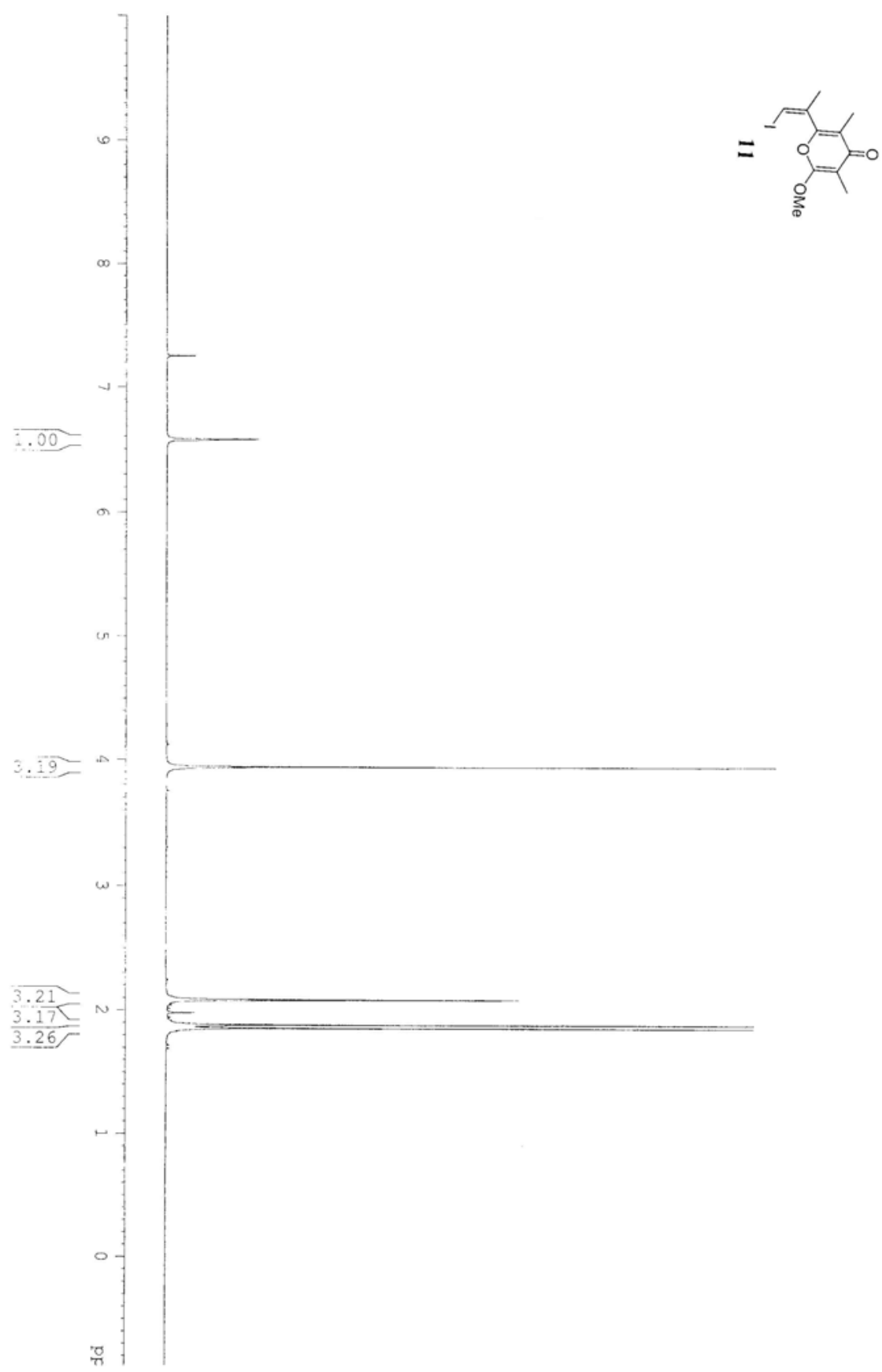




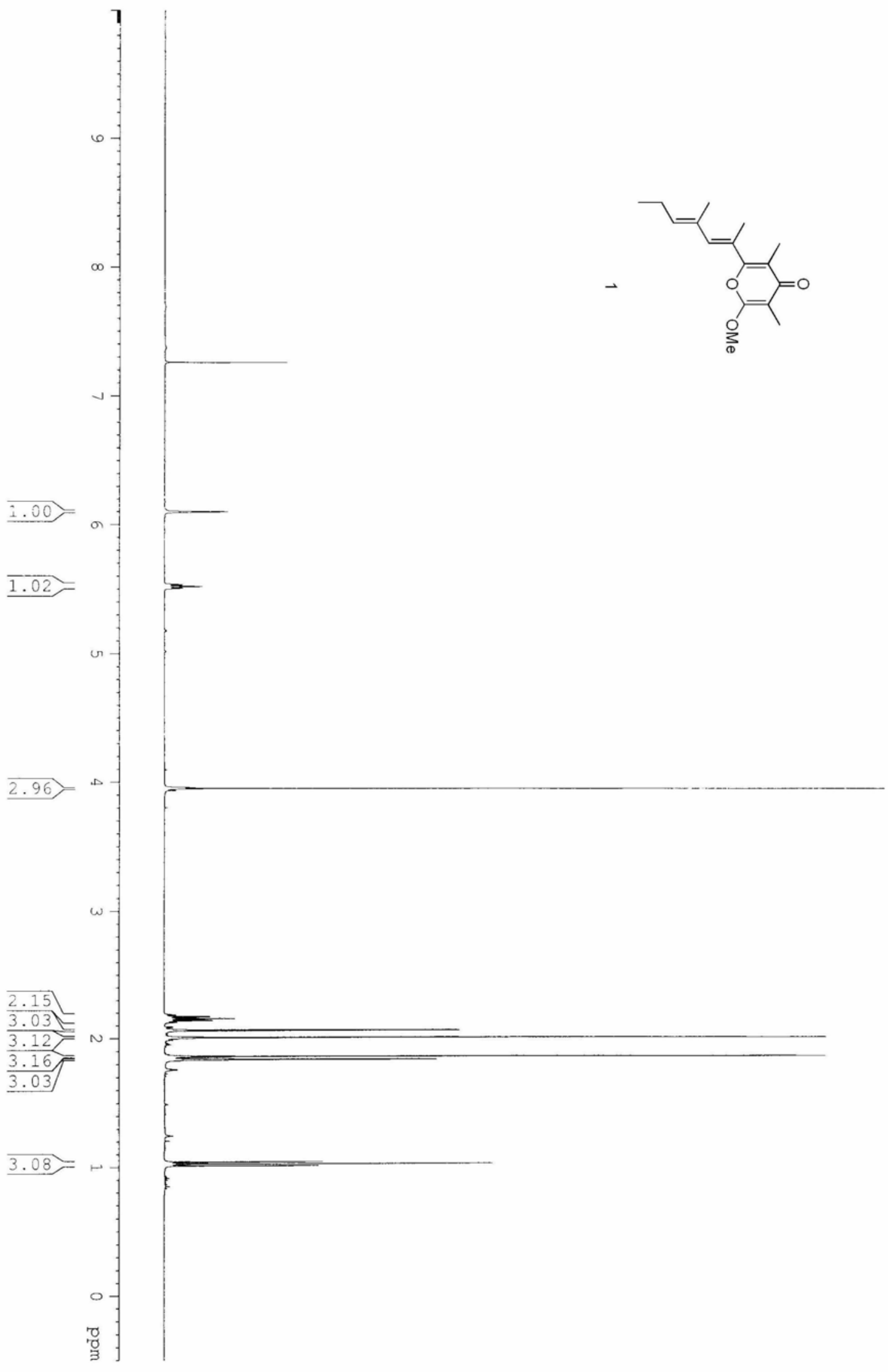




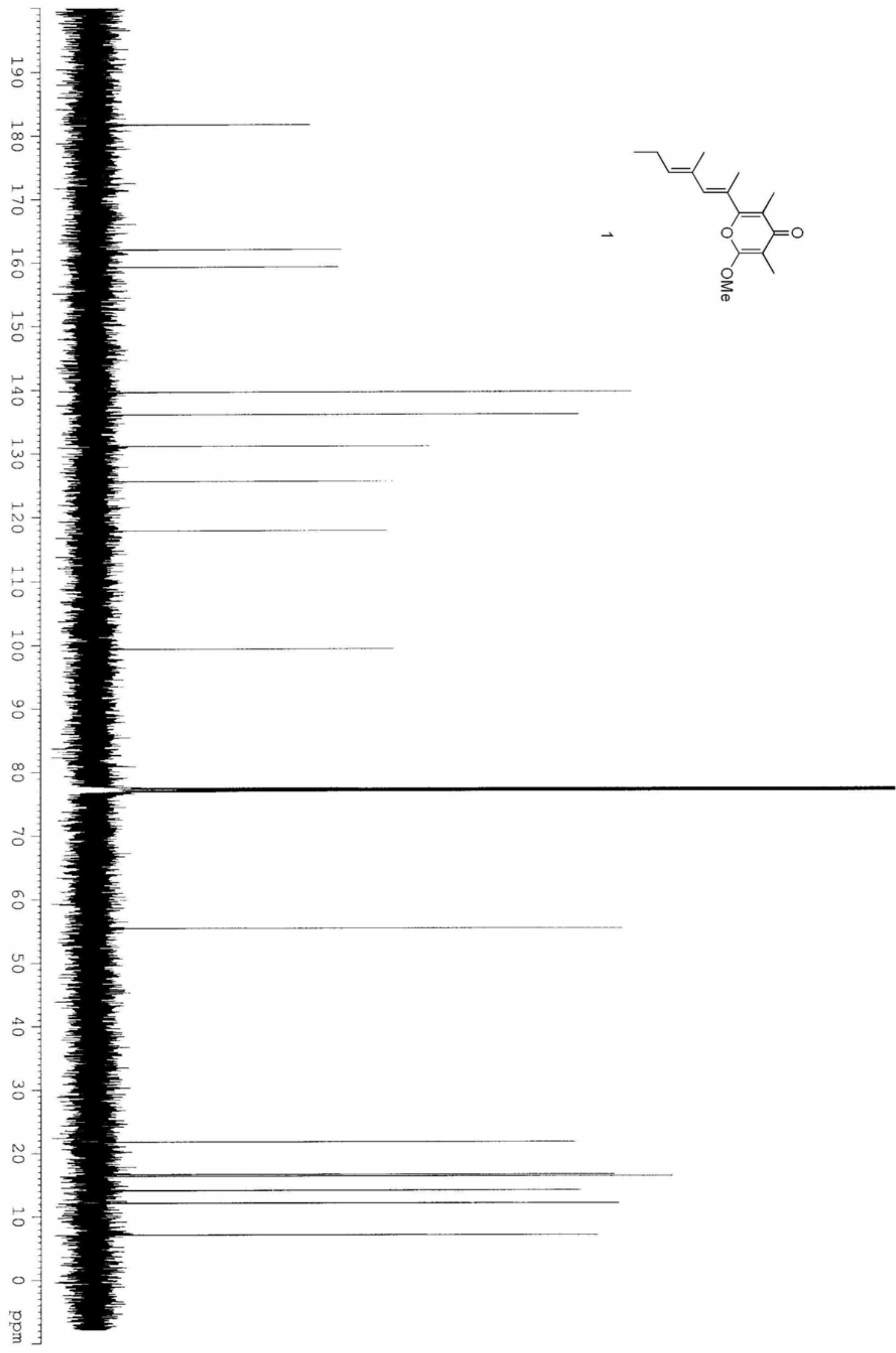




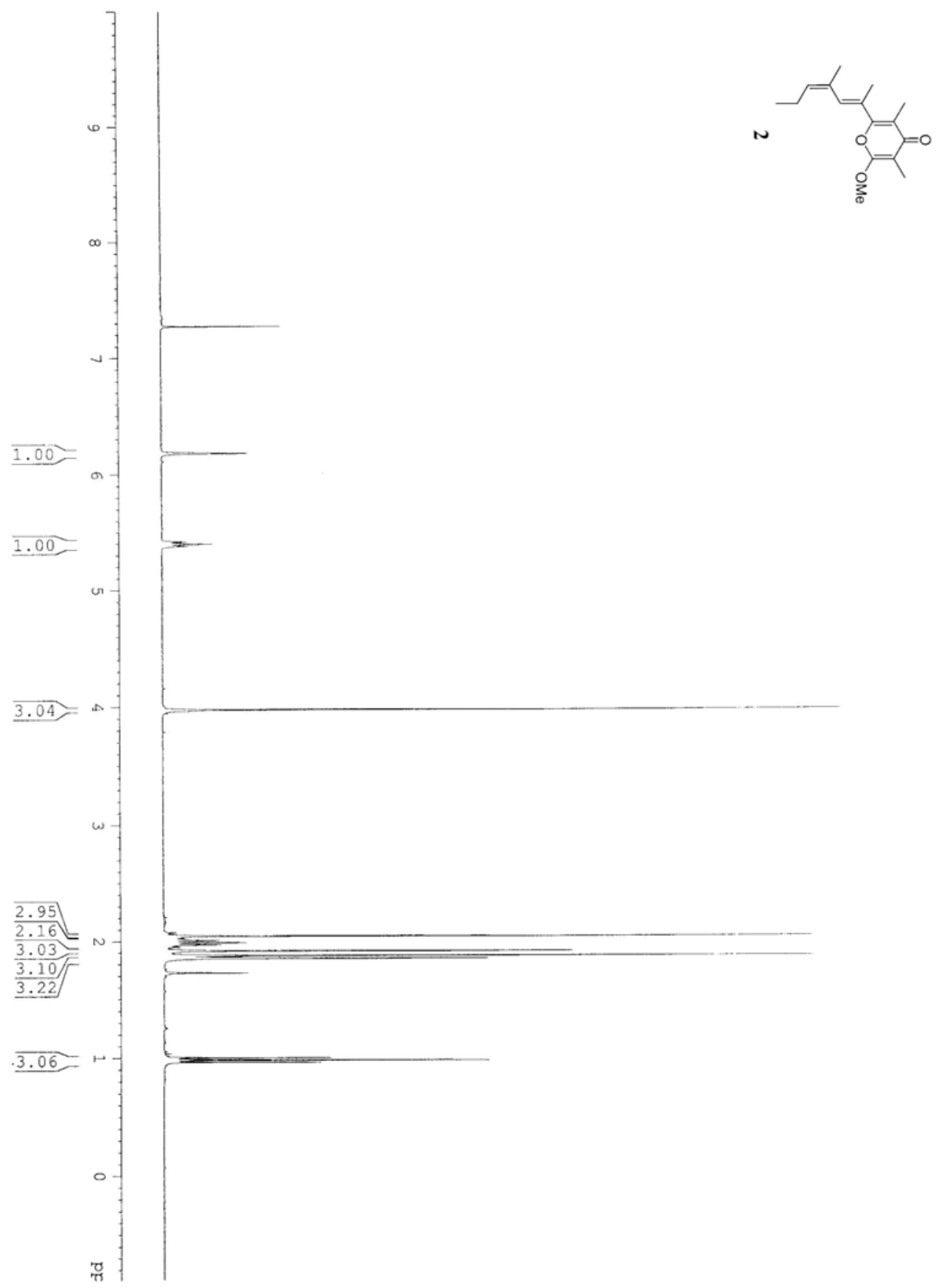




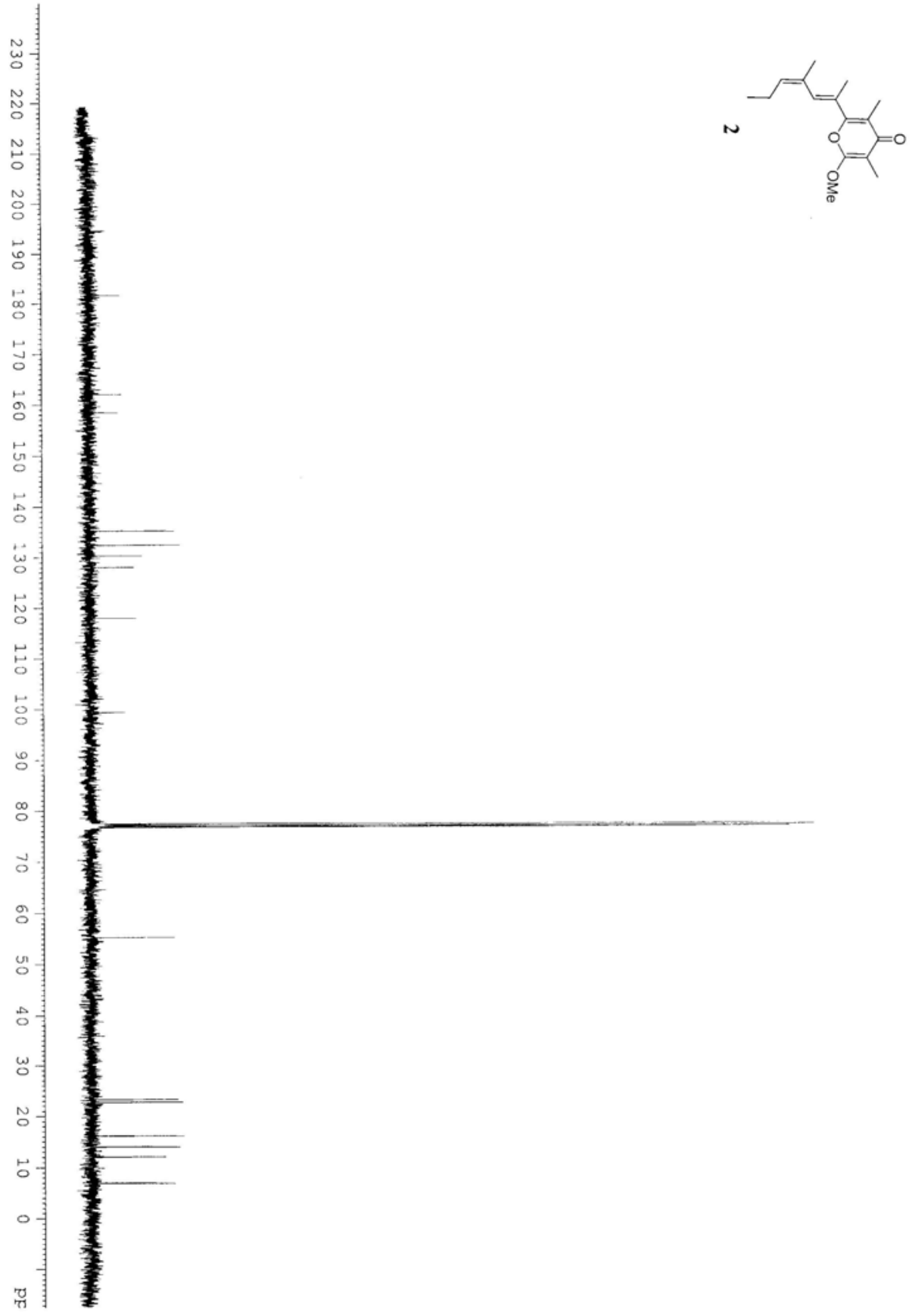




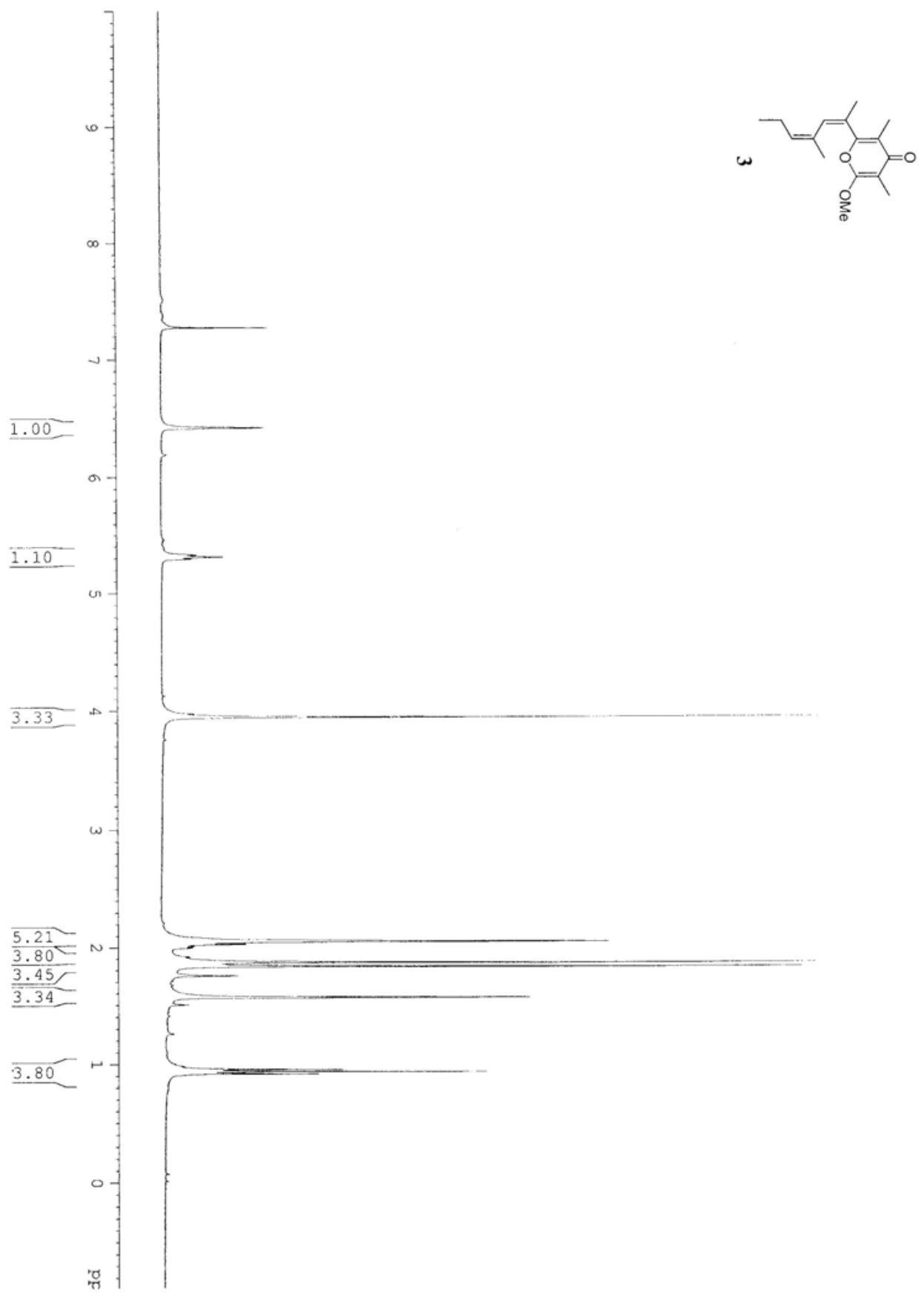




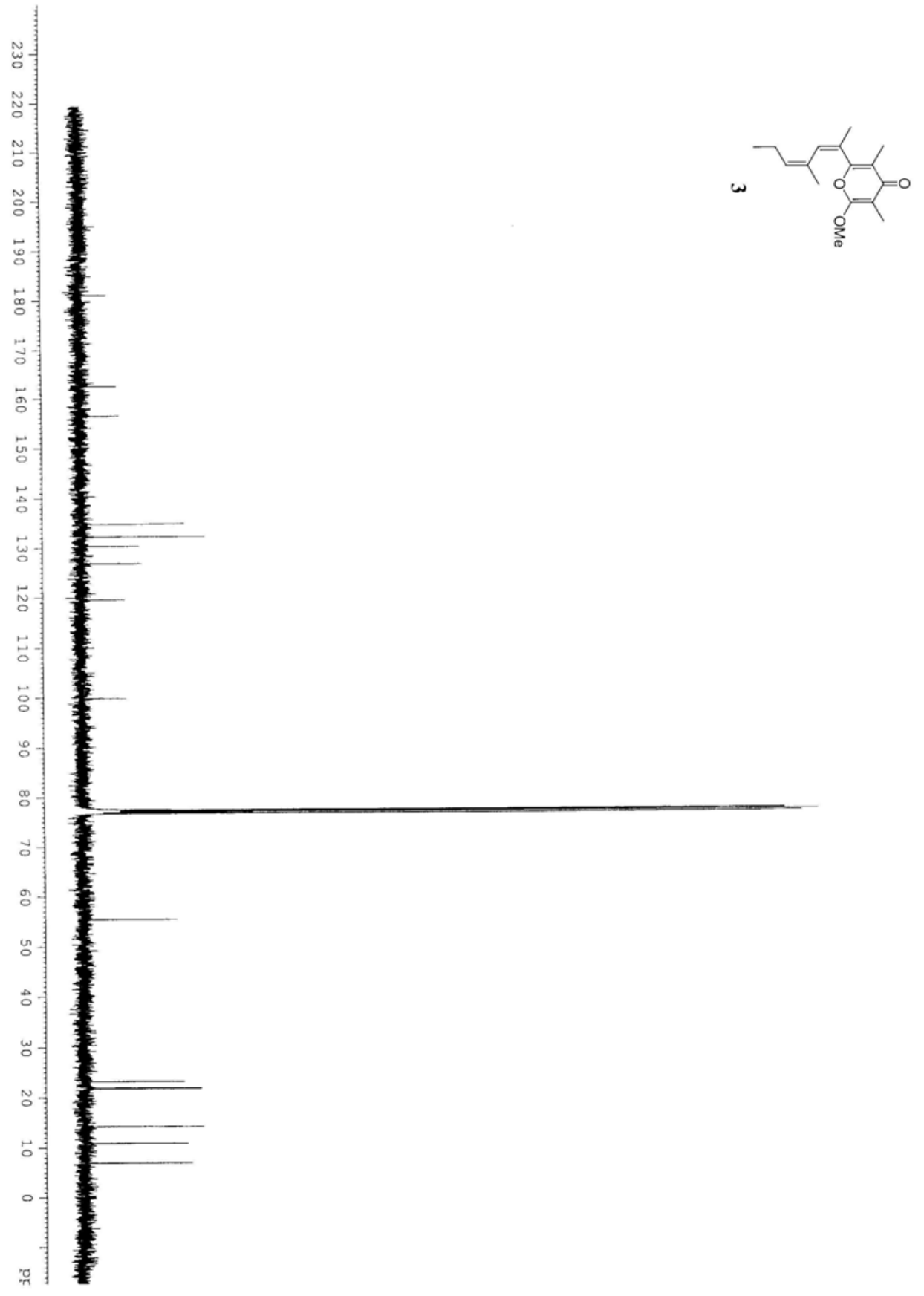




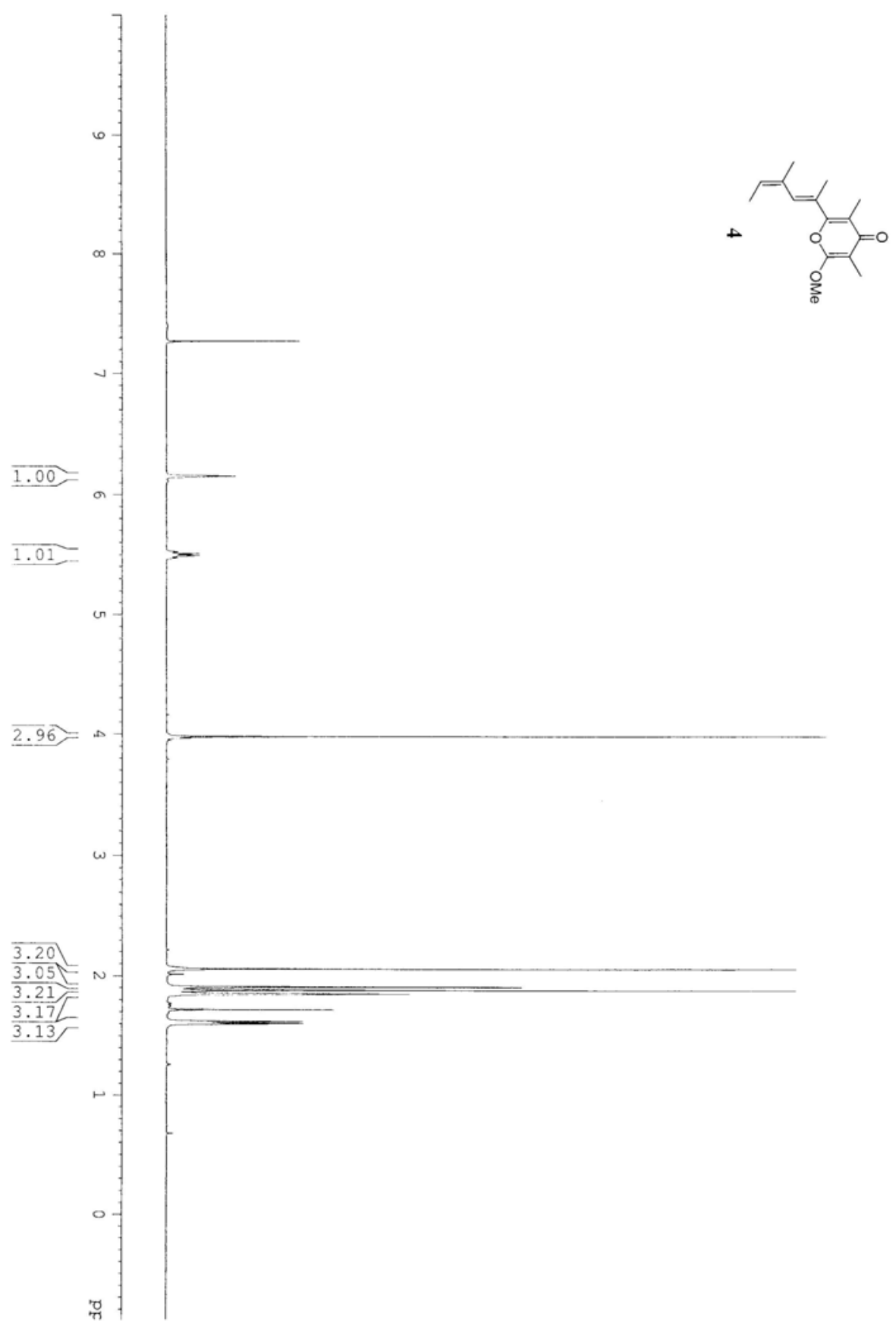




$$
\text { | }
$$




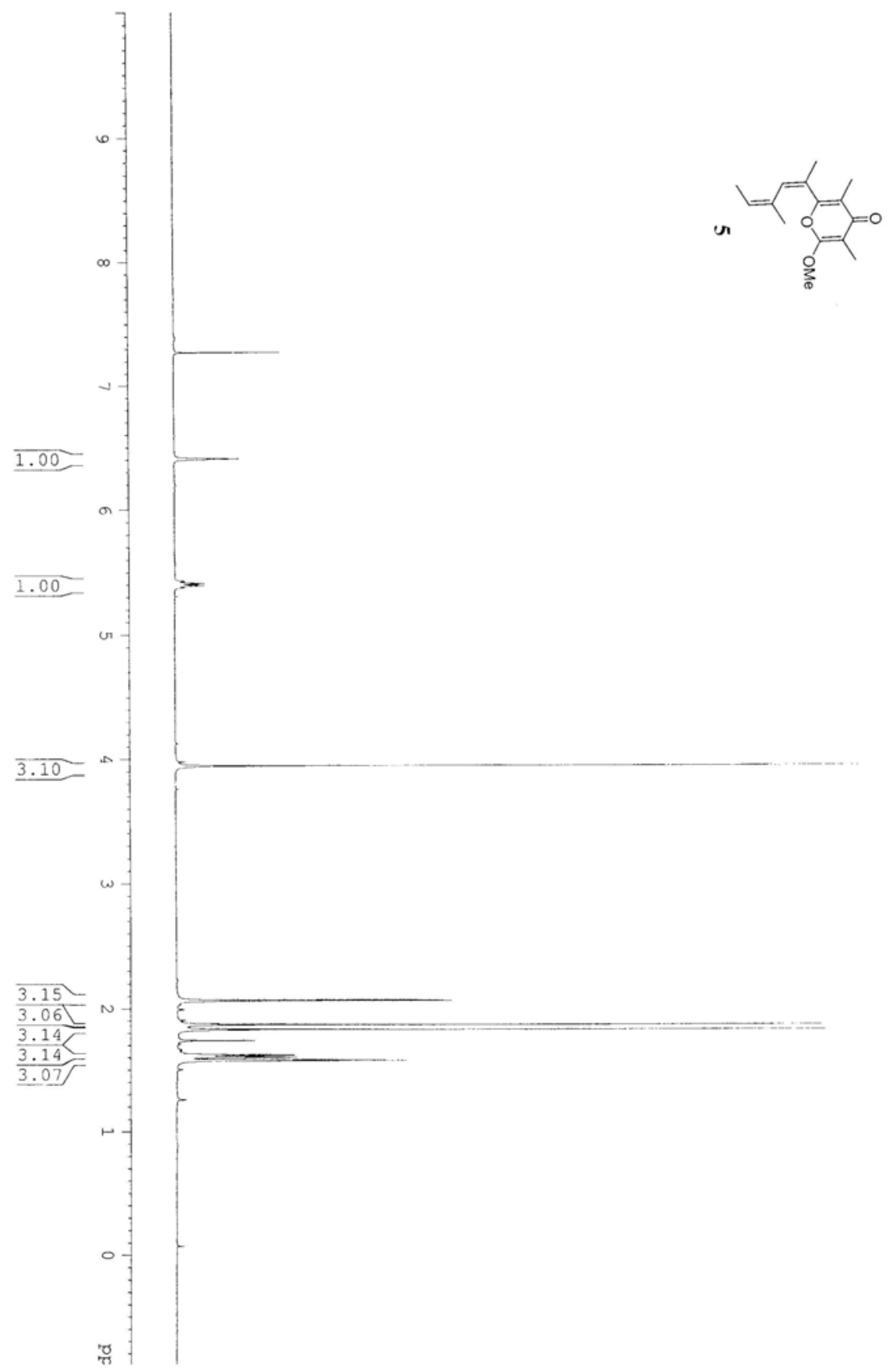




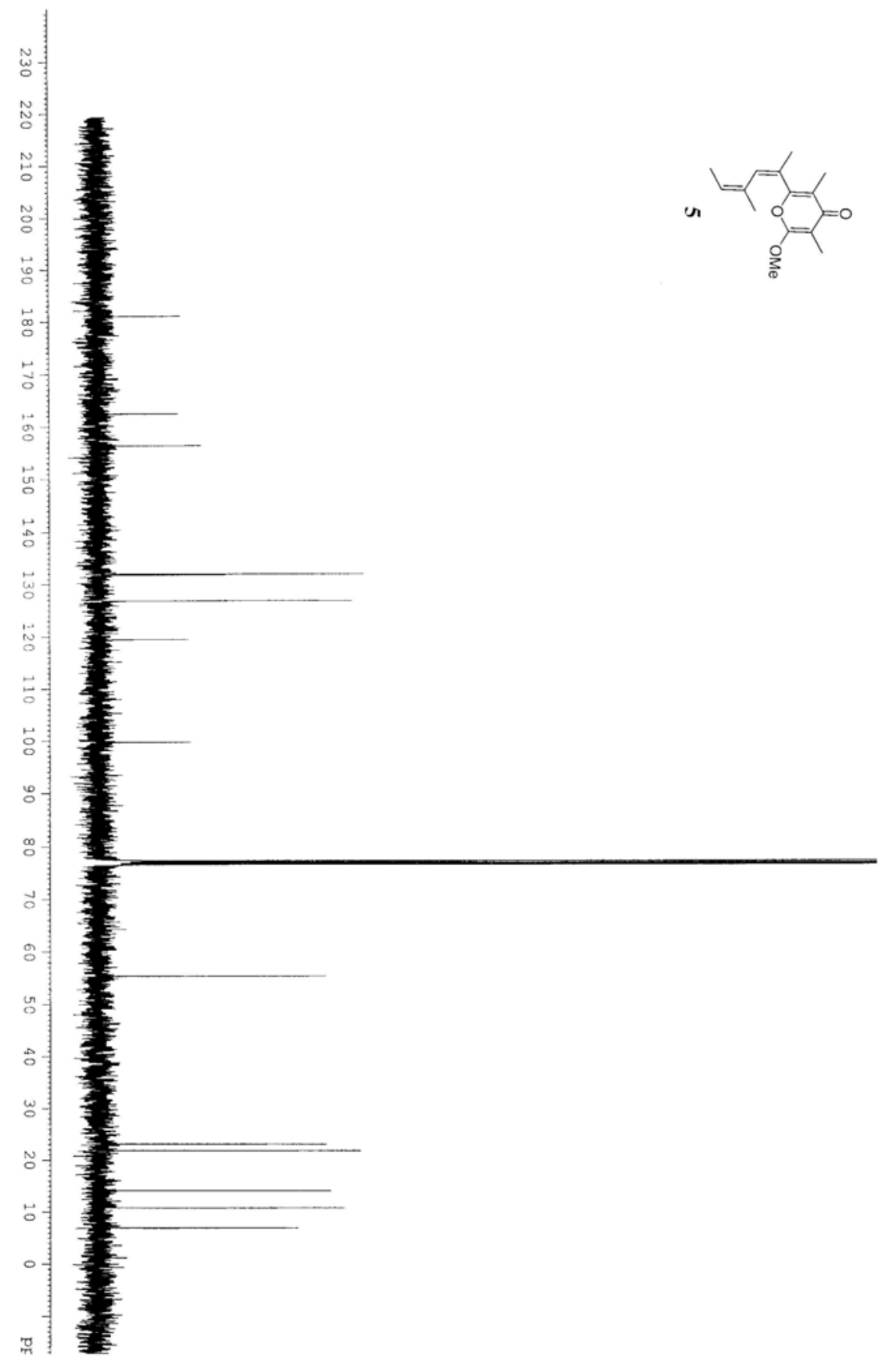




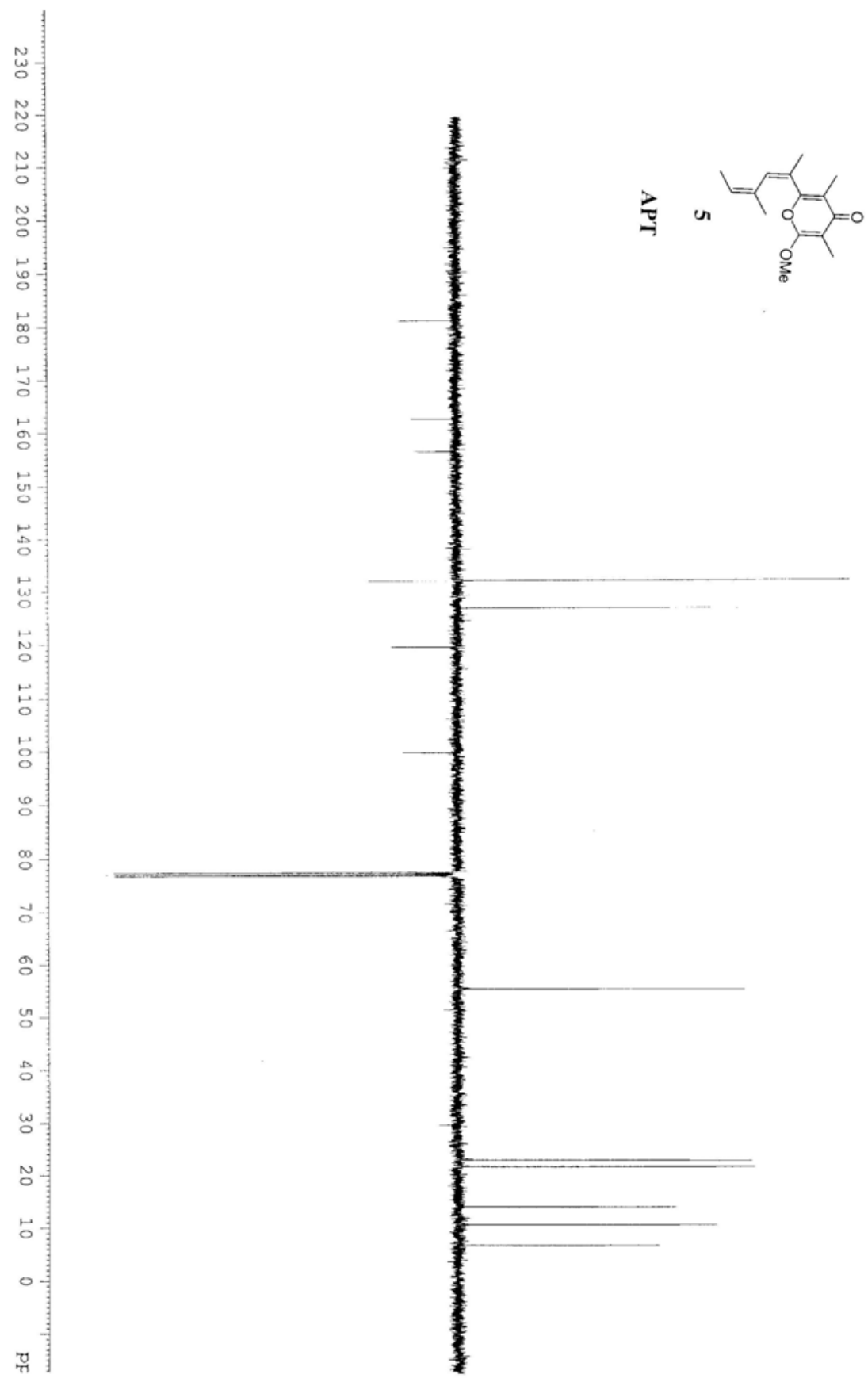

